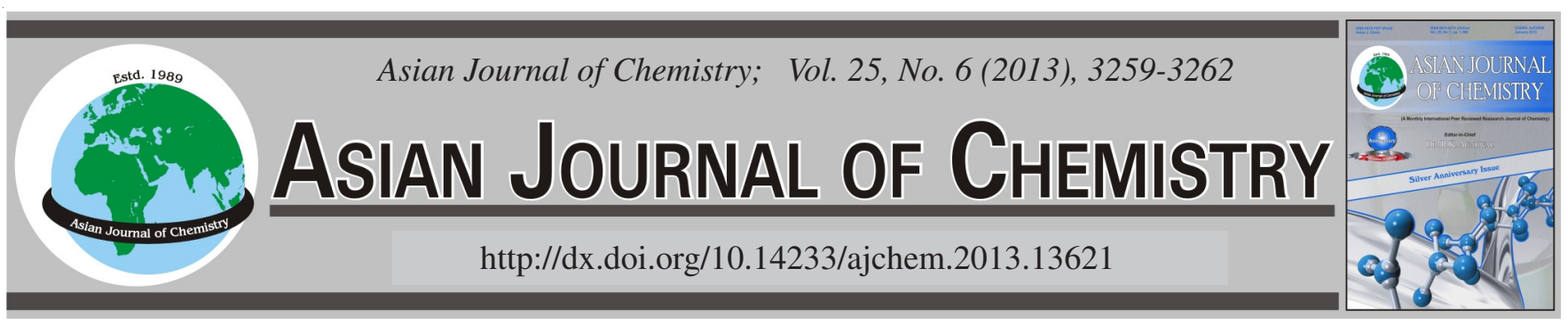

\title{
Solid-Phase Synthesis of the Hainantoxin-III and Its Antinociception Analysis in the Acid-Induced Writhing Responses of Mice
}

\author{
Yu Liu*, Zhe Wu, Xiaohong Xun, Dongsong Nie and Yang Xiang
}

College of Chemistry and Chemical Engineering, Hunan Institute of Science and Technology, Yueyang 414006, P.R. China

*Corresponding author: E-mail: hiliu2000@yahoo.com.cn

(Received: 18 February 2012;

Accepted: 17 December 2012)

AJC-12561

\begin{abstract}
Hainantoxin-III (HNTX-III), a neurotoxic peptide from the Chinese spider Ornithoctonus hainana Liang, was synthesized by Fmoc solidphase peptide synthesis method. The solid-phase carrier was rink amide resin. The synthetic peptide was cleaved from the resin and deprotected by a $90 \%$ trifluoroacetic acid solution containing $5 \%$ thioanisole, $3 \%$ ethanedithiol and $2 \%$ anisole. The product was purified by RP-HPLC and then incubated with glutathione and reduced glutathione to form the correct disulfide bond linkages. The refolded synthetic peptide was purified by RP-HPLC and then co-eluted with native hainantoxin-III. The results indicated that the synthetic hainantoxin-III has the same chemical and conformational structure as those of the native hainantoxin-III from the spider. In this study, we use the synthetic toxin to investigate the antinociceptive effect of intramuscular injected hainantoxin-III in acetic acid writhing reflex test in mice and to compare its efficacy with morphine. The study confirms that intramuscular injection of hainantoxin-III, like morphine, has antinociceptive effect in the mice model of inflammatory pain, suggesting that it may be a potential drug in clinical control of inflammatory pain.
\end{abstract}

Key Words: Hainantoxin-III, Synthesis, Antinociception.

\section{INTRODUCTION}

Hainantoxin-III (HNTX-III) is a 33-residue blocking of sodium channels that was isolated from the venom of the Chinese bird spider Ornithoctonus hainana Liang $^{1}$ which has recently been identified as a typical inhibitor cystine knot motif. The toxin has a high proportion of basic residues and it is cross-linked by three conserved intramolecular disulfide bond. The linkage pattern of disulfide bridges in HNTX-III is I-IV, II-V and III-VI as assigned by partial reduction and sequence analysis and the $\mathrm{C}$-terminal ends amidated (Fig. 1). It has been shown to specifically inhibit the neuronal tetrodotoxin-sensitive (TTX-S) voltage-gated sodium channels (VGSCs) in adult rat dorsal root ganglion (DRG) neurons with $\mathrm{IC}_{50}$ values of 30 $\mathrm{nM}$ and seem to interact with neurotoxin receptor site 1 through a mechanism quite similar to that of TTX without affecting the activation and inactivation kinetics. The HNTX-III did not affect $\mathrm{Ca}^{2+}$ channels (both high voltage activated and low voltage activated types) nor tetrodotoxin-resistant VGSCs ${ }^{2,3}$.

In this paper, we report the solid-phase chemical synthesis of HNTX-III with the Fmoc method. The objectives of this work are to provide sufficient materials to enable extensive studies of this toxin. We also report here the investigation of the optimal conditions for the correct formation of the three bonds of the synthesized HNTX-III. The results of the chemical and co-elution analysis and the electro-physiological testing of the final product are also reported. The purpose of the toxin synthesis was therefore to investigate the antinociceptive effect of intramuscular administrated HNTX-III in acetic acid writhing test in mice and to compare its efficacy with morphine.

NH2-G-C-K-G-F-G-D-S-C-T-P-G-K-N-E-C-C-P-N-Y-A-C-S-S-K-H-K-W-C-K-V-Y-L-CONH

Fig. 1. Amino acid sequence and disulfide bond arrangements in HNTXIII

\section{EXPERIMENTAL}

DTT, trifluoroacetic acid (TFA), glutathione (GSSG) and reduced glutathione (GSH) were products of Sigma. PALPEG-PS resin, Fmoc-AA-OH, HOBt and TBTU were from ABI Ltd; acetonitrile and methanol (HPLC grade) were purchased from Linhai Chemical. All other reagents were of analytical grade.

Peptide synthesis, folding and purification: HNTX-III was synthesized starting from a poly(ethylene glycol) polystyrene resin equipped with a peptide amide linker (Fmocpeptide amide linker-polyethylene glycol-polystyrene (PAL-PEG-PS) amide resin, Applied Biosystems) on an automatic peptide synthesizer (PerSeptive Biosystems) using an Fmoc/tert-butyl strategy and HOBt/TBTU/NMM coupling 
method. All amino acids were purchased from Chemassist Corp. and side chains were protected as follows: trityl for Asn, Cys and His; tert-butyl ester for Asp and Glu; tert-butyl ether for Ser, Thr and Tyr; 2,2,4,6,7-pentamethyldihydrobenzofuran5-sulfonyl for Arg; butoxycarbnyl for Trp and tert-butyloxycarbonyl for Lys. Peptide synthesis was accomplished on a $0.10 \mathrm{mmol}$ scale. The terminal Fmoc group was removed by treatment with $1: 4$ piperidine/N,N-dimethylformamide $(\mathrm{v} / \mathrm{v})$. After completion of synthesis, the peptide was cleaved from the resin with simultaneous removal of side chain protective groups by treatment with reagent $\mathrm{K}(82.5 \%$ trifluoroacetic acid, $5 \%$ double distilled water, $5 \%$ phenol, $5 \%$ thioanisole and $2.5 \%$ ethanedithiol) for $2 \mathrm{~h}$ at room temperature. The resin was then filtered and the free peptide was precipitated in cold ether at $4{ }^{\circ} \mathrm{C}$. After centrifugation and washing once with cold ether, the peptide was dissolved in $20 \%$ acetic acid and lyophilized. The reduced peptides were purified by semipreparative reverse-phase HPLC using a 42 min linear gradient of $10-50 \%$ eluent B $(0.1 \%$ trifluoroacetic acid in acetonitrile $)$ in eluent $\mathrm{A}(0.1 \%$ trifluoroacetic acid in double distilled water $)$ over $42 \mathrm{~min}$ on a column at $2 \mathrm{~mL} / \mathrm{min}$ flow rate. Peaks were analyzed by analytical HPLC and more than $95 \%$ pure fractions were pooled and lyophilized. The linear peptides were oxidized with glutathione and purified using the method recently described by Zhu et al. ${ }^{4}$. The molecular weights of the reduced peptides or oxidized peptides were checked by matrix-assisted laser desorption/ionization time-of-flight (MALDI-TOF) mass spectrometry on a Voyager-DETM STR BiospectrometryTM workstation. For HNTX-III, the measured molecular mass corresponded to the predicted value within 1.0 unit, consistent with the correctness of the sequence and the complete removal of all side chain protection groups.

Co-elution synthetic HNTX-III and native HNTX-III by HPLC: The mixture, $1 \mathrm{mg}$ synthetic HNTX-III and $1 \mathrm{mg}$ native HNTX-III, was analyzed by HPLC. Elution was performed with a linear gradient from 10-50 \% buffer B (Buffer A: water with $0.1 \%$ trifluoroacetic acid; buffer B: acetonitrile, $0.1 \%$ trifluoroacetic acid) over $42 \mathrm{~min}$.

Electro-physiological experiments: The whole cell patch clamp experiments were made from rat DRG neurons as described previously ${ }^{5}$. Rat DRG neurons were acutely dissociated and maintained in a short term primary culture using the method described by $\mathrm{Hu}$ and $\mathrm{Li}^{6}$. In brief 30 day-old adult Sprague-Dawley rats of either sex were killed by decapitation and the dorsal root ganglia were isolated quickly from the spinal cord. Then they were transferred into Dulbecco's modified eagle's medium containing trypsin $(0.5 \mathrm{mg} / \mathrm{mL}$, type III, Sigma), collagenase $(1.0 \mathrm{mg} / \mathrm{mL}$, type IA, Sigma) and DNase $\left(0.1 \mathrm{mg} / \mathrm{mL}\right.$, type III, Sigma) to incubate at $34{ }^{\circ} \mathrm{C}$ for $0.5 \mathrm{~h}$. Trypsin inhibitor $(1.5 \mathrm{mg} / \mathrm{mL}$, type II-S, Sigma) was used to terminate enzyme treatment. The DRG cells were transferred into $35 \mathrm{~mm}$ dishes (Corning, Sigma) with the culture medium and incubated in a $\mathrm{CO}_{2}$ incubator $\left(5 \% \mathrm{CO}_{2}, 95 \%\right.$ air at $37^{\circ} \mathrm{C}$ ) for $1-4 \mathrm{~h}$ before the patch clamp experiment.

Patch clamp experiments were performed at room temperature $\left(20-25{ }^{\circ} \mathrm{C}\right)$ under the whole cell patch clamp configuration. Patch pipettes (2-3 $\mu$ m diameter) were pulled from borosilicate glass capillary tubing by using a two-step vertical puller (PC-10, Narishige, Olypmus) and heat-polished with a microforge (MF-900, Narishige). Patch pipettes with resistances of 1.0-2.0 megaohms were used. The series resistance was compensated 65-70\% and linear capacitative and linear leakage currents were digitally subtracted by a $\mathrm{P} / 4$ procedure. Sodium currents were filtered at $10 \mathrm{kHz}$ and digitized at $3 \mathrm{kHz}$ with an EPC-9 patch clamp amplifier (HEKA Electronics, Germany). Experimental data were collected and analyzed by using the program Pulse/Pulsefit 8.0 (HEKA Electronics, Lambrecht/Pfalz, Germany). The patch pipettes contained $135 \mathrm{mM} \mathrm{CsF}, 10 \mathrm{mM} \mathrm{NaCl}, 5 \mathrm{mM}$ HEPES with the $\mathrm{pH}$ adjusted to 7.0 with $1 \mathrm{M} \mathrm{CsOH}$. The external bathing solution contained $30 \mathrm{mM} \mathrm{NaCl}, 5 \mathrm{mM} \mathrm{CsCl}, 25 \mathrm{mM}$ Dglucose, $1 \mathrm{mM} \mathrm{MgCl}$, $1.8 \mathrm{mM} \mathrm{CaCl}_{2}, 5 \mathrm{mM}$ HEPES, $20 \mathrm{mM}$ tetraethylammonium chloride, $70 \mathrm{mM}$ tetramethylammonium chloride with the $\mathrm{pH}$ adjusted to 7.40 with $1 \mathrm{M}$ tetraethylammonium hydroxide. Peptide toxin was dissolved in external solution and about a $10 \mu \mathrm{L}$ volume was applied by pressure injection with a microinjector (IM-5B, Narishige). All chemical reagents were purchased from Sigma.

Acetic acid writhing reflex test: Adult Kunming mice (either sexes, weighting 20-22 g) were used for this behavioural test. Animals were provided by the Hunan slack-kinda experimental animal limited company and use of the animal was reviewed and approved by the Animal Care and Use Committee. Mice were divided into three groups of eight each. Group one received control, group one received synthetic HNTX-III and the remaining group received morphine. 15 min later, $0.2 \mathrm{~mL}$ of $0.6 \%$ acetic acid was injected into the abdominal cavity. After 5 min lag time, the number of abdominal contractions (writhing movement) was observed for $15 \mathrm{~min}$ and record. Percentage inhibition of writhing movement was then calculated from the values obtained.

Statistics: Data were expressed as means \pm SEM and examined by one-way analysis of variance (ANOVA) followed by Dunnett's multiple comparison test. A $p$-value of less than 0.05 was considered significant.

\section{RESULTS AND DISCUSSION}

Peptide synthesis and characterization: Solid-phase synthesis of HNTX-III, using Fmoc protected amino acids and HOBt/TBTU coupling, yielded a major product as revealed by reverse-phase HPLC analysis and MALDI-TOF mass spectrometry. The purified reduced peptide was folded/oxidized in buffer $0.1 \mathrm{M}$ tris-HCl, $0.1 \mathrm{M} \mathrm{NaCl}, \mathrm{pH} 7.4$, containing $5 \mathrm{mM}$ GSH, $0.5 \mathrm{mM} \mathrm{GSSG}$ for $24 \mathrm{~h}$ at room temperature. Purified products were homogeneous in analytical reverse-phase HPLC (Fig. 2) and their masses (3615 Da, $3609 \mathrm{Da}$, for reduced HNTX-III, oxidized HNTX-III, respectively, Fig. 3) were in good accordance with the theoretical masses.

Bioactivity analysis of the synthesized HNTX-III: The biological activities of the synthesized HNTX-III were studied by testing their capacity to inhibit the TTX-S sodium channels in rat DRG neurons. The dose-response curves shown in Fig. 4 illustrate the block of sodium channels at peptide concentrations ranging from $1 \mathrm{nM}$ to $10 \mu \mathrm{M}$. The $\mathrm{IC}_{50}$ values of 34.2 $\mathrm{nM}$ were estimated for the synthesized HNTX-III. The value was approximately similar to that of native HNTX-III. 

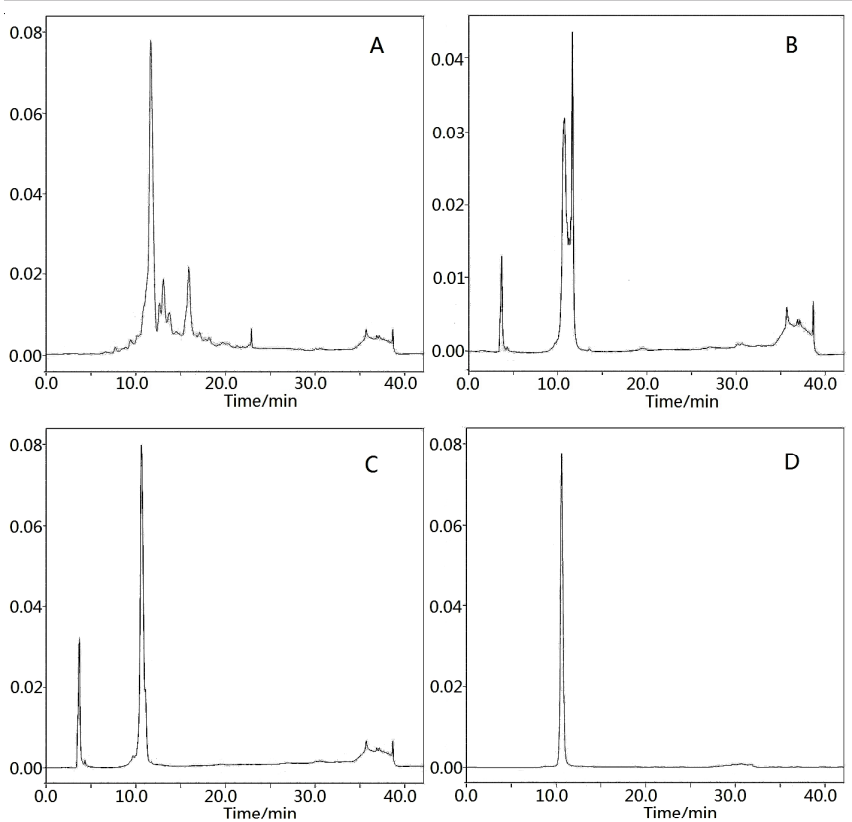

Fig. 2. Analytical reversed-phase HPLC chromatograms of (A) crude product; (B) reduced synthetic HNTX-III after oxidation 5 min; (C) reduced synthetic HNTX-III after oxidation $24 \mathrm{~h}$; (D) the mixture of synthesized and native HNTX-III at an equal amount
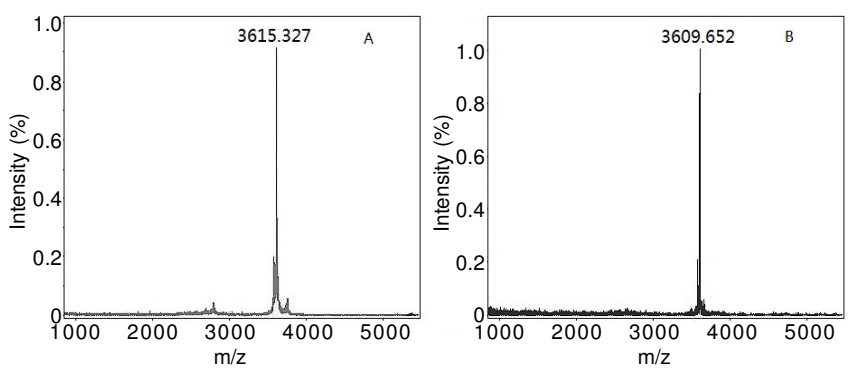

Fig. 3. MALDI-TOF mass spectra of HNTX-III (A) reduced synthetic HNTX-III); (B) oxidation synthetic HNTX-III. The mass spectrometry results show that the oxidized synthetic HNTX-III was formed three-disulfide bridged

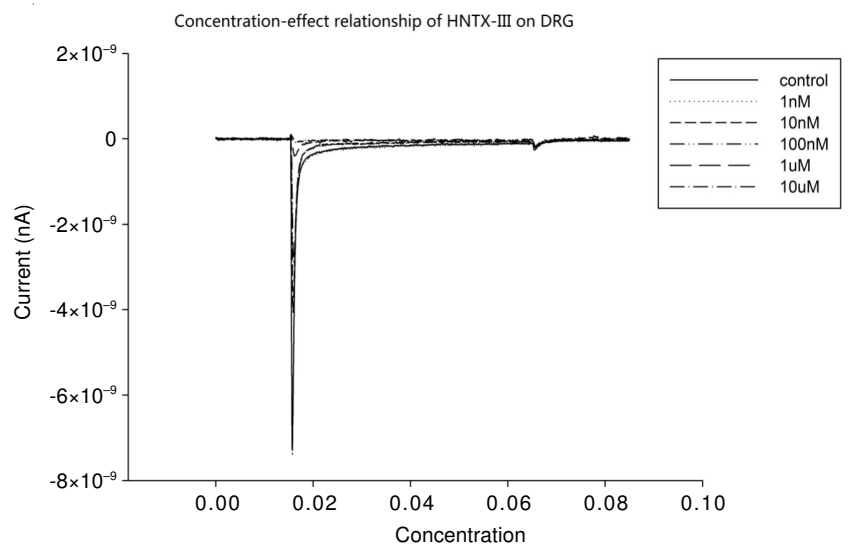

Fig. 4. Inhibition potency of HNTX-III on TTX-S Na ${ }^{+}$currents in rat DRG cells. Dose-dependent inhibition of peptide on TTX-S Na ${ }^{+}$currents. Every data point (mean $\pm \mathrm{SE}$ ), which came from three to eight cells, shows current relative to control. The value was approximately similar to that of native HNTX-III $\left(\mathrm{IC}_{50}=34.2 \mathrm{nM}\right)$

HNTX-III significantly reduced writhings and stretchings induced by the acetic acid (Fig. 5). The protective effect was dose dependent with $18.06 \%(p<0.05)$ reduction observed for $20.0 \mu \mathrm{g} / \mathrm{kg}$ and $65.16 \%(p<0.01)$ seen for $100 \mu \mathrm{g} / \mathrm{kg}$ dose. The HNTX-III had an $\mathrm{ED}_{50}$ of $65.0 \mu \mathrm{g} / \mathrm{kg}$ with a confidence limit of $20-100 \mu \mathrm{g} / \mathrm{kg}$. Morphine (a centrally acting analgesic) had $57.42 \%(p<0.01)$ inhibition with $50 \mu \mathrm{g} / \mathrm{kg}$. From the analgesic effect, the analgesic effect of $100 \mathrm{mg} / \mathrm{kg}$ of HNTX-III was equal to the analgesic effect of the $50 \mathrm{mg} / \mathrm{kg}$ of morphine.

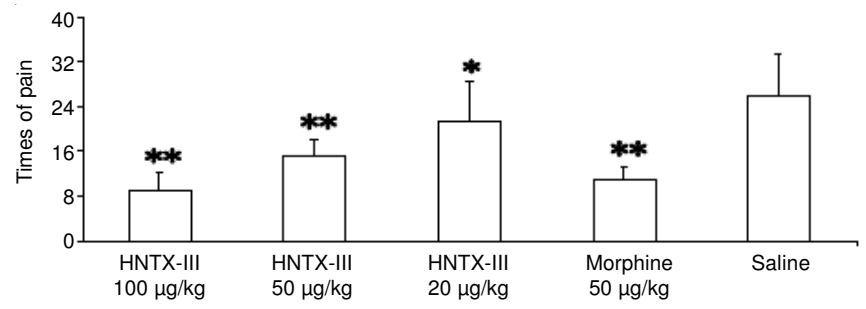

Fig. 5. Column graph shows the influence of HNTX-III on mouse writhing reflex induced by acetic acid. Group differences were considered statistically significant at $p<0.05 . * * p<0.01 ; * p<0.05$

The acetic acid-induced writhing reaction in mice, described as a typical model for inflammatory pain, has long been used as a screening tool for evaluation of analgesic or antiinflammatory agents ${ }^{7}$. Major transmission pathway of the inflammatory pain has been documented as the pathway comprising peripheral polymodal receptors around small vessels that signal the central nervous system via sensory afferent C-fibers that enter the dorsal horn ${ }^{8}$. Bradykinin ${ }^{9}$, substance $\mathrm{P}^{10}$ and prostaglandins ${ }^{11}$ were documented as mediators involved in the writhing responses induced by acetic acid, acetylcholine or phenylquinone. Berkenkopf reported PGI2 as a major prostanoid, based on the detection of 6-keto-PGF1a in the exudates of mice undergoing an acetic acid-induced writhing response. The Yuri Ikeda's study is the first to demonstrate the contribution of the VR1 receptor to the acidinduced writhing response ${ }^{12}$. The analgesic potential of the HNTX-III was shown by acetic acid test to be effective. HNTXIII has been shown to specifically inhibit the mammal neuronal TTX-S sodium channels in DRG cells ${ }^{2}$, meaning TTX-S sodium channels was contributed to the acid-induced writhing response and HNTX-III is a good pain drug lead.

$\omega$-Conotoxin MVIIA as a new non-morphine analgesics, respectively, in 2004 and 2005 the USFDA and the European Union (EC) approved as a treatment for chronic pain analgesic drugs (Ziconotide) listed. However, the antinociception induced by $\omega$-conotoxin MVIIA were companied with motor dysfunction, such as whole body shaking, circling, ataxia and tail wiggling and so on characteristic body shaking behaviours as described previously ${ }^{13-15}$ during the $1 \mathrm{~h}$ observation period. These side-effects became more evident with the doses of $\omega$ conotoxin MVIIA increasing. In contrast, HNTX-III did not have those side-effects at the doses of $10-4000 \mu \mathrm{g} / \mathrm{kg}$. On the other hand, $\omega$-conotoxin MVIIA need to intrathecal injection, HNTX-III can be intramuscular injected. It is very beneficial on drug delivery.

In summary, the neurotoxic peptide HNTX-III has been synthetic by solid phase method with Fmoc method. The synthesized toxin has been proved to have the same chemical and three-dimensional structure and to possess the same 
biological activity. The study confirms that intramuscular injection of HNTX-III, like morphine, has antinociceptive effect in the mice model of acid-induced inflammatory pain, suggesting that it may be a potential drug in clinical control of pain. As the new TTX-S sodium channel antagonists, perhaps HNTX-III possess more extensive applicative outlook for the treatment of pain, hyperalgesia and allodynia in clinical than classical drugs and ziconotide.

\section{ACKNOWLEDGEMENTS}

This work was supported by the National "973" Program of China (No. 2010CB529800) and the Opening Fund of Key Laboratory of Protein Chemistry and Developmental Biology of the Ministry of Education, Hunan Normal University, and the Science and Technology Project of Hunan Province (2012SK3042).

\section{REFERENCES}

1. $\quad$ S.P. Liang, X.J. Peng, R.H. Huang and P. Chen, Life Sci. Res., 3, 299 (1999).
2. Y.C. Xiao and S.P. Liang, Eur. J. Pharmacol., 477, 1 ( 2003).

3. Y.C. Xiao, J.Z Tang, W.J. Hu, C. Maertens, J. Tytgat and S. Liang, J. Biol. Chem., 280, 12069 (2005).

4. Q. Zhu, S. P.Liang, L. Martin, S. Gasparini, A. Menez and C. Vita, Biochemistry, 41, 11488 (2002).

5. Z. Liu, J. Dai, Z. Chen, W. Hu, Y. Xiao and S. Liang, Cell Mol. Life Sci., 60, 972 (2004).

6. H.Z. Hu and Z.W. Li, J. Physiol., 501, 67 (1997).

7. R. Vinegar, J.F. Truax, J.L. Selph and P.R. Johnston, Handbook of Experimental Pharmacology, Springer: Verlag, Berlin, Vol. 50/II, p. 208 (1997).

8. T. Kumazawa, K. Mizumura, H. Koda and H. Fukusako, J. Neurophysiol., 75, 2361 (1996).

9. C.R. Correa, D.J. Kyle, S. Chakraverty and J.B. Calixto, Br. J. Pharmacol., 117, 552 (1996).

10. V.M. Goettl and A.A. Larson, Brain Res., 780, 80 (1998).

11. S. Moncada, S.H. Ferreira and J.R. Vane, Eur. J. Pharmacol., 31, 250 (1975).

12. Y. Ikeda, A. Ueno, H. Naraba and S. Oh-ishi, Life Sci., 69, 2911 (2001).

13. A.B. Malmberg and T.L. Yaksh, Pain, 60, 83 (1995).

14. K.K. Jain, Expert. Opin. Investig. Drugs, 9, 2403 (2000).

15. R.D. Penn and J.A. Paice, Pain, 85, 291 (2000). 